\title{
LETTER
}

Acute lymphoblastic leukemia

\section{B-cell leukemia transdifferentiation to macrophage involves reconfiguration of DNA methylation for long-range regulation}

\author{
Alberto Bueno-Costa ${ }^{1} \cdot$ David Piñeyro $\mathbb{D}^{1} \cdot$ Marta Soler $^{1} \cdot$ Biola M. Javierre ${ }^{1} \cdot$ Helena Raurell-Vila ${ }^{2}$. \\ Marc Subirana-Granés ${ }^{2} \cdot{\text { Lorenzo Pasquali } \mathbb{D}^{1,2,3} \cdot \text { Jose A. Martinez-Climent }}^{4,5} \cdot$ Manel Esteller (D) ${ }^{1,5,6,7}$
}

Received: 4 July 2019 / Revised: 18 October 2019 / Accepted: 3 November 2019 / Published online: 12 November 2019

(c) The Author(s) 2019. This article is published with open access

\section{To the Editor:}

Hematopoiesis is a highly regulated process that, starting from hematopoietic stem cells (HSCs) with self-renewal capacity in the adult human bone marrow, is able to generate all different types of mature blood cells. The classical view of hematopoiesis defines binary branching points from these HSCs that segregate lineages and direct differentiation to terminally differentiated functional cell types [1]. However, the described hierarchical model can be complemented with the emerging data that suggest the existence of hematopoietic stem and progenitor cells with a continuum of transitory differentiation stages, including cells with early

Supplementary information The online version of this article (https:// doi.org/10.1038/s41375-019-0643-1) contains supplementary material, which is available to authorized users.

$\triangle$ Manel Esteller

mesteller@carrerasresearch.org

1 Josep Carreras Leukaemia Research Institute (IJC), Badalona, Barcelona, Catalonia, Spain

2 Program of Predictive and Personalized Medicine of Cancer (PMPPC), Endocrine Regulatory Genomics Laboratory, Department of Endocrinology and Nutrition, Germans Trias i Pujol University Hospital and Research Institute (IGTP), Badalona, Catalonia, Spain

3 CIBER de Diabetes y Enfermedades Metabólicas Asociadas (CIBERDEM), Barcelona, Spain

4 Division of Hematological Oncology, Center for Applied Medical Research (CIMA), University of Navarra, Pamplona, Spain

5 Centro de Investigacion Biomedica en Red Cancer (CIBERONC), 28029 Madrid, Spain

6 Institucio Catalana de Recerca i Estudis Avançats (ICREA), Barcelona, Catalonia, Spain

7 Physiological Sciences Department, School of Medicine and Health Sciences, University of Barcelona (UB),

Barcelona, Catalonia, Spain lineage priming that generate distinct blood cell types according to the physiological or pathological environment [2]. In this context, there are increasing data of hematopoietic plasticity and cell lineage conversion, particularly in leukemogenesis. Examples of transdifferentiation include B-cell lymphomas that can transform to histiocytic/dendritic cell sarcoma, erythroid/megakaryocytic lineages changing to granulomonocytic-like lineage upon use of a histone demethylase LSD1 inhibitor or B-ALL (acute lymphoblastic leukemia) patients that evaded CD19-directed antibody therapy (blinatumomab) by undergoing myeloidlineage switch. Related to the latter scenario, lineage switching has also been reported as a cause of antigen loss in chimeric antigen receptor T-cell therapies, where B-ALL patients transdifferentiate in their relapse as acute myeloblastic leukemia in response to the initial CD19-directed immunotherapy [3]. Due to the central role of epigenetics, particularly DNA methylation, in the successful generation of differentiated blood cell types and its plasticity during lineage specification [4], we wondered about its function in hematopoietic transdifferentiation, a largely unexplored field.

Our studied model of transdifferentiation is a welldefined experimental system that converts B cells into macrophages. Following initial work that demonstrated that normal murine B-cell precursors as well as mature antibody-producing $\mathrm{B}$ cells can be induced by $\mathrm{C} / \mathrm{EBP} \alpha$ to transdifferentiate into functional macrophages [5], a murine cellular model was established of pre-B cells containing a fusion of $\mathrm{C} / \mathrm{EBP} \alpha$ with the estrogen receptor hormone binding domain $(\mathrm{C} / \mathrm{EBP} \alpha \mathrm{ER})$ that converts them to macrophage-like cells upon $17 \beta$-estradiol exposure [6]. We have recently translated this model to human B-lymphoma and leukemia cell lines that can be induced by $\mathrm{C} / \mathrm{EBP} \alpha$ to transdifferentiate into functional macrophages [7]. Importantly, primary human BCR-ABL1(+) B-ALL cells could also be induced to reprogram into macrophage-like cells by transient expression of $\mathrm{C} / \mathrm{EBP} \alpha[8]$. To explore the changes 
that the DNA methylome undergoes upon transdifferentiation, we have herein applied this experimental system. Thus, we have analyzed the human precursor B-ALL cell line RCV-ACH transfected with the transgene C/EBP $\alpha E R$, thereafter termed BLaER1, upon 17 $\beta$-estradiol-mediated transdifferentiation at seven timepoints $(0,3,12,24,48,72$, and $168 \mathrm{~h}$ ) using a comprehensive DNA methylation microarray that interrogates more than $850,000 \mathrm{CpG}$ sites (Supplementary Fig. 1a and Supplementary Methods). DNA methylation data are available on the GEO repository under accession number GSE132845. We have observed a significant change in the methylation status of $251 \mathrm{CpG}$ sites during the transdifferentiation process ( $p$-value $<0.05$ and $\mathrm{CpG} B$-value change $\geq 0.66$ ) (Supplementary Table 1 and Supplementary Methods). Most strikingly, all except one $(250$ of $251,99.6 \%)$ were hypomethylation changes (Fig. 1a and Supplementary Fig. 1a). In this regard, these hypomethylation events occurred in the context of downregulation of the DNA methyltransferases DNMT1 and DNMT3B, but not DNMT3A, in our transdifferentiation model (Supplementary Fig. 2). The DNA methylation pattern of the endpoint of transdifferentiation (BlaER1 at 168 h) for these sites mimicked the $\mathrm{CpG}$ methylation status of naive macrophages (Fig. 1a and Supplementary Table 1). According to genomic distribution of the identified $\mathrm{CpG}$ sites, 141 CpGs (56.2\%) had an associated gene, whereas 110 CpGs $(43.8 \%)$ were in regions of the genome without any annotated gene (Fig. 1b).

Due to our interest in epigenetic modifications that can actively contribute to the transdifferentiation phenomenon and the classical view of the impact of DNA methylation on transcription, we first studied the $141 \mathrm{CpG}$ sites that are annotated as having an associated gene in relation with the available expression levels of the corresponding gene [9]. We identified $41 \mathrm{CpG}$ sites (29.1\%), corresponding to 39 genes (Supplementary Table 2), for which the methylation status significantly correlated with the expression of the associated gene: in most cases the $\mathrm{CpG}$ hypomethylation event was linked to gene expression (32 of 39, 82\%), whereas only in a minority of cases was demethylation associated with gene repression (7 of 39, 18\%) (Supplementary Table 2). Using data mining (Supplementary Methods), we observed that these $41 \mathrm{CpG}$ sites were in binding sites for 80 transcription factors (Supplementary Fig. 3a). Gene ontology analysis using a hypergeometric test to find biological processes overrepresented in our set of transcription factors (Supplementary Methods) demonstrated, in addition to global regulatory networks, an enrichment in the "immune system development," "cell fate commitment," and "leukocyte differentiation" categories (Supplementary Fig. 3b), including homeobox proteins related to leukemia biology (such as MEIS1, HOXA2, and HOXC11) and lymphoid and myeloid-differentiation- associated programs (such as FOXC1, GATA2, and SPI1). We proceeded further to validate two candidate genes detected as undergoing $\mathrm{CpG}$ demethylationassociated reactivation for our multiomic approaches in the transdifferentiation model: the interleukin-1 receptor antagonist (IL1RN) and integrin alpha X (ITGAX), both genes that are almost exclusively expressed in macrophages $[10,11]$. The methylation changes of the identified $\mathrm{CpG}$ sites associated with IL1RN and ITGAX were further confirmed by bisulfite genomic sequencing of multiple clones and bisulfite pyrosequencing (Supplementary Fig. 4 and Supplementary Methods). Quantitative real-time PCR (Supplementary Methods) demonstrated lack of expression for both transcripts in the initial B-ALL cells in the absence of 17b-estradiol treatment and a strong gain of expression at the end of the transdifferentiation process in the macrophage-like cells (Fig. 1c). The assessment of protein levels for IL1RN (western blot) and ITGAX (cell cytometry) (Supplementary Methods) obtained the same expression patterns (Fig. 1c). The use of the demethylating agent 5-aza-2'-deoxycytidine in BLaER1 cells induced IL1RN and ITGAX expression (Supplementary Fig. 5), supporting the role of $\mathrm{CpG}$ methylation in gene silencing. Most importantly, $17 \beta$-estradiol treatment of the original naive pre-B-ALL leukemia cells RCH-ACV, which were not transfected with the transgene C/EBP $\alpha E R$ and thus do no transdifferentiate, maintained the gene silencing of IL1RN and ITGAX (Fig. 1c). These data support the role of a wave of DNA demethylation that, acting locally in the CpG-associated genes, confers macrophage identity to the original pre-B-ALL cells.

Strikingly, our analysis workflow showed, as described above (Fig. 1b), that among the differentially methylated CpGs in our transdifferentiation model, 110 CpGs (43.8\%) were located in genome contexts without any genes in their vicinity. Thus, in these cases, it is possible that a candidate regulatory event of those CpGs occurs in the $3 \mathrm{D}$ organization of the human genome. In this regard, regulation at long-range distance can occur due to the formation of "loops" in the DNA that, for example, collocate enhancer or silencer sequences and minimal promoters. DNA methylation patterns at enhancers are relevant to the determination of cell identity [12] and their aberrant $\mathrm{CpG}$ methylation profile has been observed in human cancer [13], including hematological malignancies. Thus, we investigated how many of these nongene associated CpGs were located in distant regulatory regions, taking advantage of our available Promoter Capture Hi-C (PCHi-C) data in macrophages [14]. We also included in our analyses the $141 \mathrm{CpG}$ sites with associated genes due to the possibility that, in addition to regulating genes in lineal proximity, these $\mathrm{CpG}$ sites could also act as long-range interactor sequences. We found that 72 of the overall 251 CpGs $(28.7 \%)$ were located in 

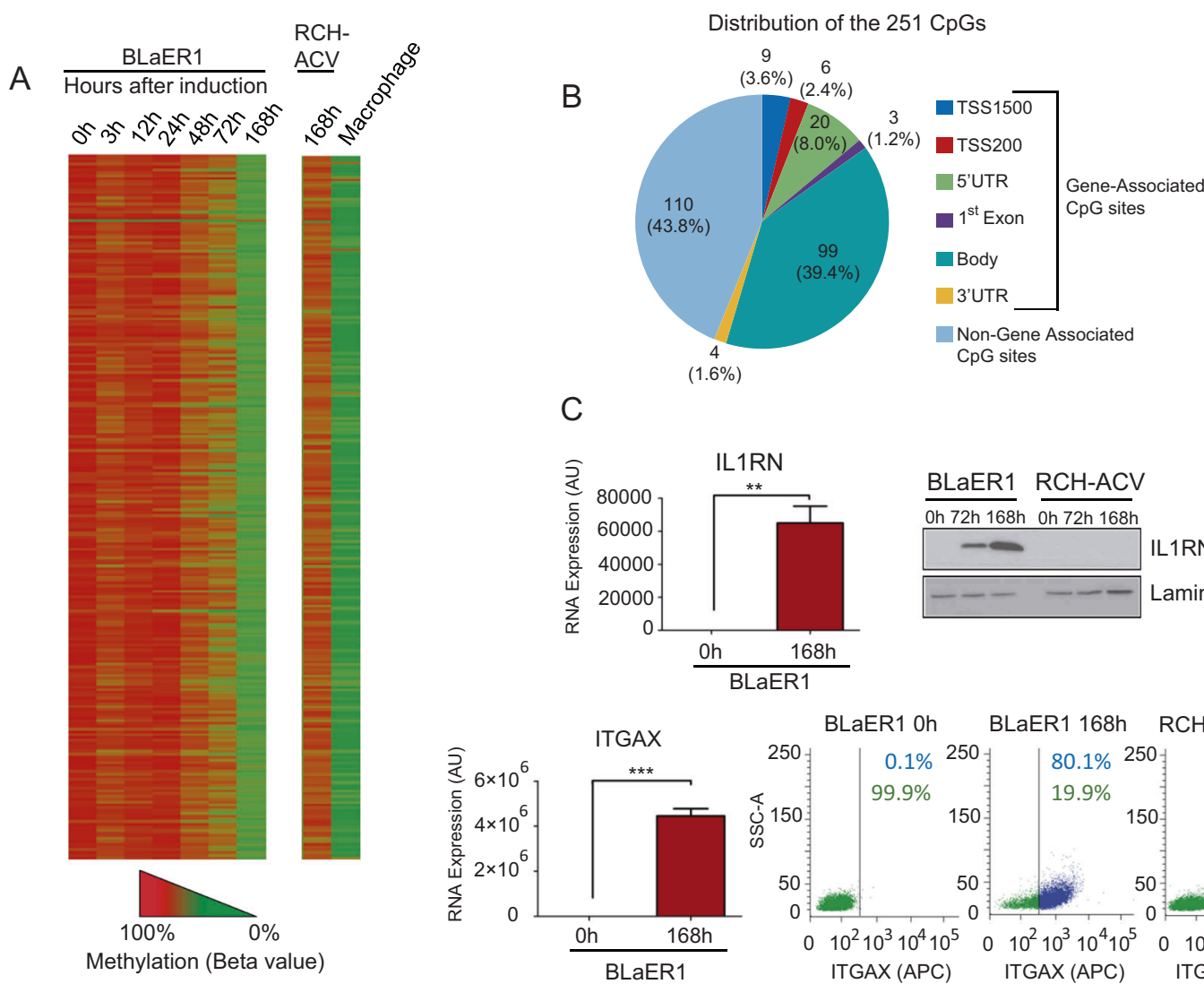

C
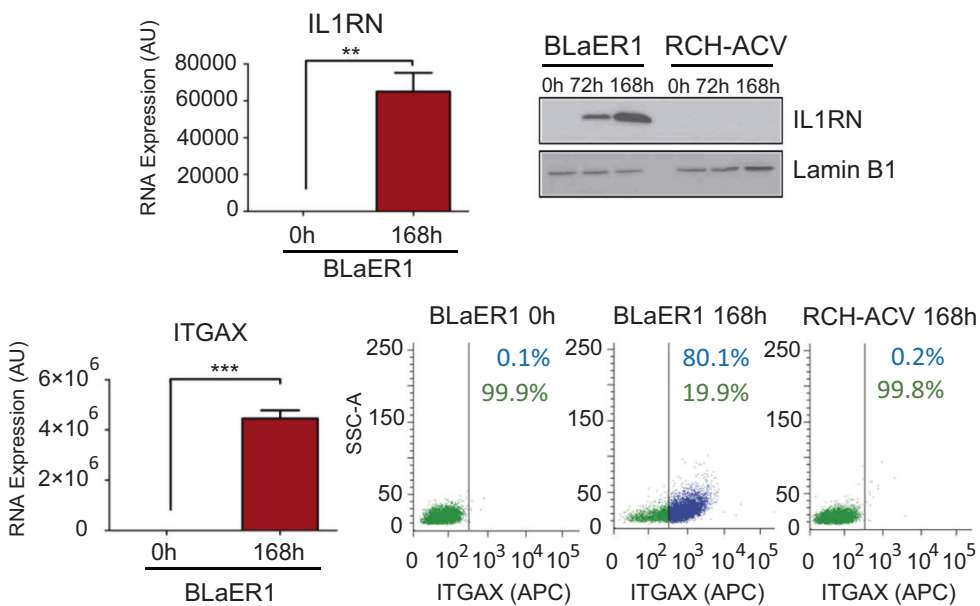

Fig. 1 DNA methylation analysis at different timepoints of B-ALL-toMacrophage transdifferentiation. a Heatmap showing the methylation state of the 251 significant hyper/hypomethylated CpGs during 7 days of transdifferentiation. RCH-ACV (treated 7 days with $17 \beta$-estradiol, IL-3, and M-CSF) and macrophage are taken as negative and positive

candidate distant regulatory regions defined by $\mathrm{PCHi}-\mathrm{C}$ in macrophages (Supplementary Fig. 1b and Supplementary Table 3) [14]. To address the functionality of the observed DNA methylation changes, we then interrogated whether the methylation status of these $72 \mathrm{CpG}$ sites had any impact on the expression of the genes whose promoters are targeted by these long-range regulatory sequences [14]. We found that the methylation status of 34 of the $72 \mathrm{CpG}$ sites $(47.2 \%)$ correlated with the expression of 52 genes that interacted with these regions (Supplementary Fig. 1b and Supplementary Table 4). Importantly, all except one (33 of 34, 97\%) were $\mathrm{CpG}$ hypomethylation changes (Supplementary Table 4). Most of the 34 PCHi-C-derived $\mathrm{CpG}$ dinucleotides represented unique interactions between one methylation site and one gene (21 of 34, 62\%) (Fig. 2a), followed by dual interactions (one $\mathrm{CpG}$ interacting with two genes, 7 of 34, 21\%), but more complex interactions were also observed (Fig. 2a). Interestingly, although many of these $\mathrm{CpG}$ sites were in nonassociated gene regions (18 of $34,53 \%$ ), we also frequently observed CpGs within associated genes (16 of $34,47 \%$ ) (Fig. 2b). It is important to highlight that the $\mathrm{CpGs}$ in the latter subset were mainly controls, respectively. $\mathbf{b}$ Pie chart showing the genomic distribution of the 251 significant CpGs. c qRT-PCR (***p $p<0.001, T$-Test) western blot and flow cytometry analysis at different timepoints of transdifferentiation of IL1RN and ITGAX, whose associated promoter CpGs are significantly demethylated during transdifferentiation

located in nonpromoter regions of the gene (11 of 16, 69\%) (Supplementary Table 4). Using data mining (Supplementary Methods), we observed that four of the PCHi-C-derived $\mathrm{CpG}$ dinucleotides contained a binding motif for the CCCTC-binding factor (CTCF), the most widely recognized protein controlling three-dimensional structures of DNA. Related to the 52 identified target genes, for 31 genes (60\%) the CpG hypomethylation event acted as an enhancer event associated with the expression of the corresponding gene, whereas for 21 genes $(40 \%)$ the demethylation event was associated with gene inactivation and can be classified as a candidate silencer sequence in our pre-B-ALL to macrophage transdifferentiation model (Supplementary Fig. 1b and Supplementary Table 4).

We experimentally validated the available expression microarray data [7] using quantitative real-time PCR for five candidate targets: three genes with $\mathrm{CpG}$ hypomethylation associated expression upon transdifferentiation (ras homolog family member G, RHOG; C-X-C motif chemokine ligand 8, CXCL8; and C-C motif chemokine receptor 1, CCR1) and two genes with $\mathrm{CpG}$ demethylation-associated expression reduction upon cell 

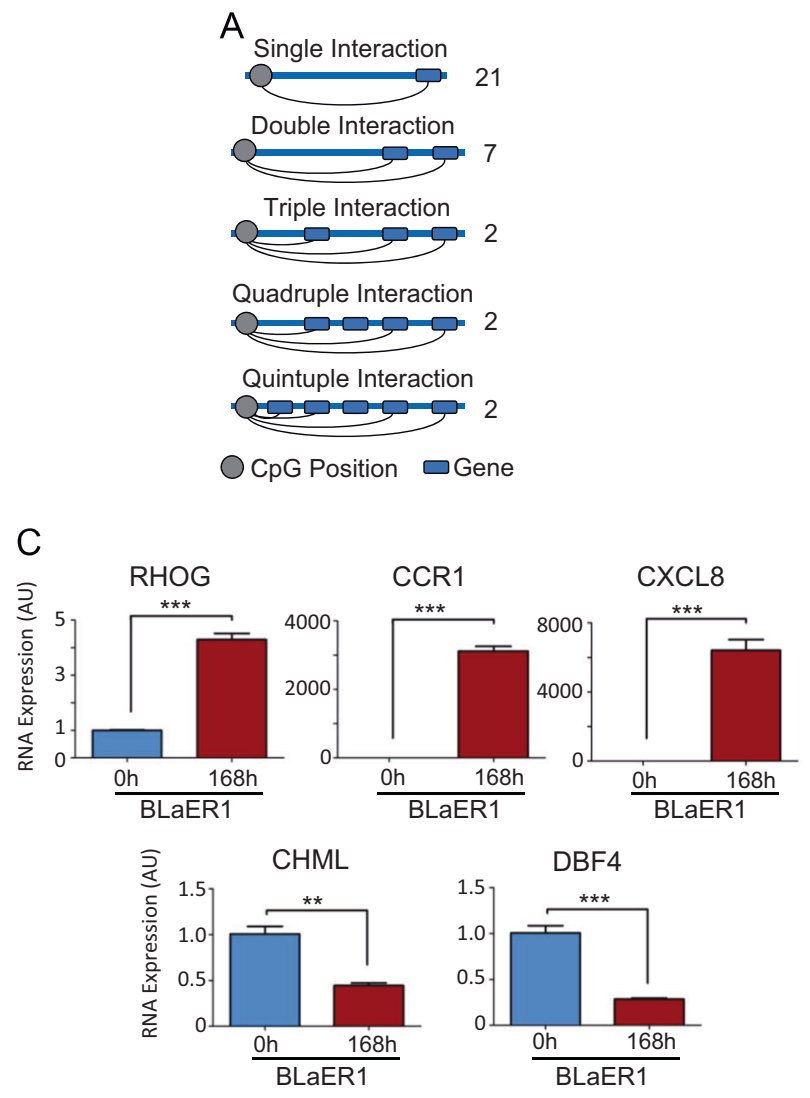

B Distribution of the 34 significant $\mathrm{CpGs}$
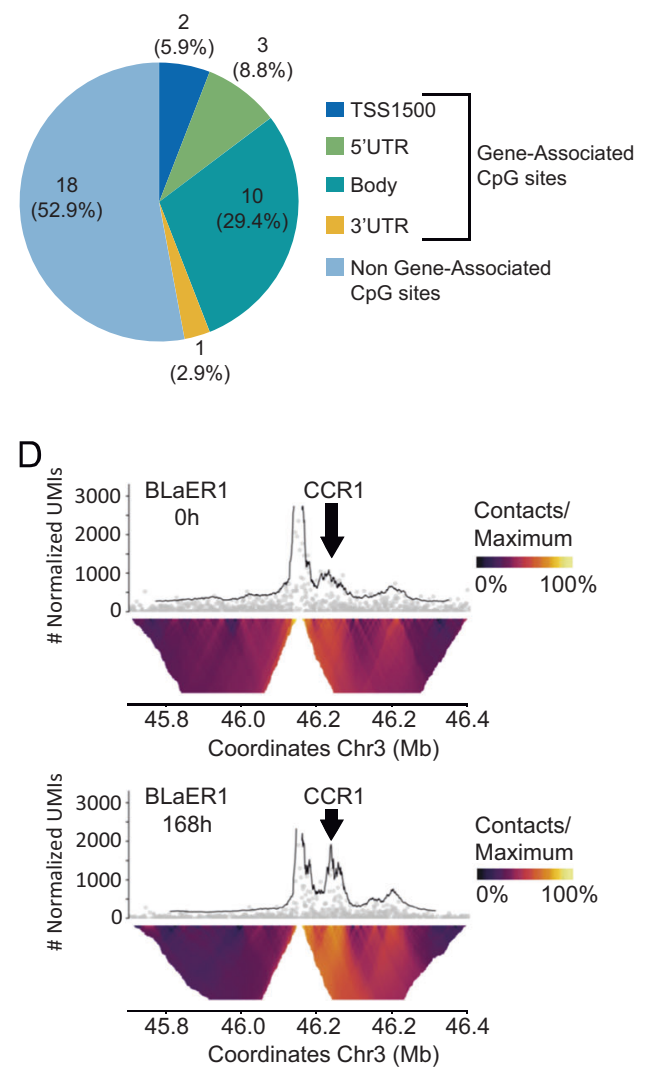

smoothed trend line of the contact profiles of the bait at time 0 (top) and at the end of BlaER1 transdifferentiation (168 h) (below). A bottom heatmap (domainogram) reports the differential mean contacts per a series of restriction enzyme fragments. The UMI-4C analysis shows that upon BlaER1 transdifferentiation the hypomethylated $\mathrm{CpG}$ containing sequence engages a strong distal chromatin contact (black arrow) with the promoter of CCR1. Of note the chromatin contact intensity for the same region was very low for the hypermethylated regulatory sequence at time $0 \mathrm{~h}$ of transdifferentiation (Chi-square adjusted $p<0.0001$ )

contact quantifications only between DNA sequences located within a $\sim 0.5 \mathrm{~Kb}$ and $\sim 1 \mathrm{Mb}$ interval [15]: among the five studied genes, only the CCR1 promoter and its putative $\mathrm{PCHi}-\mathrm{C}$ obtained $\mathrm{CpG}$-containing regulatory region fulfilled these parameters. UMI-4C data are available at the SRA repository under accession number PRJNA548887. Most importantly, we found that the CpG demethylation event occurring during transdifferentiation for the PCHi-C derived candidate long-distance regulatory sequence of CCR1 was not only associated with the expression of the gene (Fig. 2c), but also with the formation of a new loop between the CpG-containing distant regulatory region and the CCR 1 proximal promoter (Chisquare test $p<0.0001$ ) (Fig. 2d), supporting a role as an enhancer. These data highlight the relevance of DNA methylation events at distant regulatory regions to confer cell identity for both B-ALL and macrophage cells. technical limitation of UMI-4C is that it provides reliable 
In conclusion, we report that transdifferentiation events in the context of hematopoietic lineage plasticity, such as the pre-B-ALL lineage conversion to macrophage studied herein, involve DNA methylation shifts that not only affect CpG sites in lineal proximity to genes, but also incur epigenetic changes in long-range interactor sequences derived from the 3D genome architecture of the living cell. These results may help to improve our knowledge of the critical determinant for cell type specification and to understand what goes awry in hematological malignancies that, in response to pharmacological or cellular therapies, undergo lineage switching to develop resistance to the applied treatment.

Acknowledgements We thank CERCA Programme/Generalitat de Catalunya for institutional support. ABC is a fellow of the Spanish Ministry of Education and Vocational Training, under FPU contract no. FPU17/02423. ME is an ICREA Research Professor. BMJ is a Ramon y Cajal fellow (RYC-2016-19655). This work was supported by the Health Department PERIS-project no. SLT/002/16/00374 and AGAUR-projects no. 2017SGR1080, 2014SGR633, 2017SGR1033, and 2009SGR1315 of the Catalan Government (Generalitat de Catalunya); the Spanish Institute of Health Carlos III (ISCIII) project no. DTS16/00153, and CIBERONC CB16/12/00312 and CB16/12/00489; and Ministerio de Economía y Competitividad (MINECO) project nos. SAF2014-55000-R, BFU2014-57466-P, SAF2017-89673-R, SAF2017-86242-R, and SAF2015-70270-REDT cofinanced by the European Development Regional Fund, 'A way to achieve Europe' ERDF; the Cellex Foundation; and "la Caixa" Bank Foundation (LCF/ PR/GN18/51140001). We thank Dr Thomas Graf for providing the transdifferentiation model.

Author contribution Contribution: $\mathrm{ABC}$ and $\mathrm{ME}$ conceived and designed the study; $\mathrm{ABC}$ performed all experiments; $\mathrm{ABC}, \mathrm{DP}$, and BMJ analyzed multiomics data; MS performed western blots; HRV, MSG, and LP performed UMI-4C analysis; JMC provided cellular models and primary samples; and $\mathrm{ABC}$ and $\mathrm{ME}$ wrote the manuscript with contributions and approval from all authors.

\section{Compliance with ethical standards}

Conflict of interest ME is a consultant of Ferrer International and Quimatryx. The authors declare that they have no conflict of interest.

Publisher's note Springer Nature remains neutral with regard to jurisdictional claims in published maps and institutional affiliations.

Open Access This article is licensed under a Creative Commons Attribution 4.0 International License, which permits use, sharing, adaptation, distribution and reproduction in any medium or format, as long as you give appropriate credit to the original author(s) and the source, provide a link to the Creative Commons license, and indicate if changes were made. The images or other third party material in this article are included in the article's Creative Commons license, unless indicated otherwise in a credit line to the material. If material is not included in the article's Creative Commons license and your intended use is not permitted by statutory regulation or exceeds the permitted use, you will need to obtain permission directly from the copyright holder. To view a copy of this license, visit http://creativecommons. org/licenses/by/4.0/.

\section{References}

1. Chao MP, Seita J, Weissman IL. Establishment of a normal hematopoietic and leukemia stem cell hierarchy. Cold Spring Harb Symp Quant Biol. 2008;73:439-49.

2. Velten L, Haas SF, Raffel S, Blaszkiewicz S, Islam S, Hennig B, et al. Human haematopoietic stem cell lineage commitment is a continuous process. Nat Cell Biol. 2017;19:271-81.

3. Majzner RG, Mackall CL. Tumor antigen escape from CAR T-cell therapy. Cancer Discov. 2018;8:1219-26.

4. Langstein J, Milsom MD, Lipka DB. Impact of DNA methylation programming on normal and pre-leukemic hematopoiesis. Semin Cancer Biol. 2018;51:89-100.

5. Xie H, Ye M, Feng R, Graf T. Stepwise reprogramming of B cells into macrophages. Cell. 2004;117:663-76.

6. Bussmann LH, Schubert A, Vu Manh TP, De Andres L, Desbordes SC, Parra M, et al. A robust and highly efficient immune cell reprogramming system. Cell Stem Cell. 2009;5:554-66.

7. Rapino F, Robles EF, Richter-Larrea JA, Kallin EM, MartinezCliment JA, Graf T. C/EBP $\alpha$ induces highly efficient macrophage transdifferentiation of B lymphoma and leukemia cell lines and impairs their tumorigenicity. Cell Rep. 2017;19:1281.

8. McClellan JS, Dove C, Gentles AJ, Ryan CE, Majeti R. Reprogramming of primary human Philadelphia chromosome-positive $\mathrm{B}$ cell acute lymphoblastic leukemia cells into nonleukemic macrophages. Proc Natl Acad Sci USA. 2015;112:4074-9.

9. Svensson PA, Hägg DA, Jernås M, Englund MC, Hulten LM, Ohlsson BG, et al. Identification of genes predominantly expressed in human macrophages. Atherosclerosis. 2004; 177:287-90.

10. Scott CL, T'Jonck W, Martens L, Todorov H, Sichien D, Soen B, et al. The transcription factor ZEB2 Is required to maintain the tissue-specific identities of macrophages. Immunity. 2018;49: $312-25$.

11. Heinz S, Romanoski CE, Benner C, Glass CK. The selection and function of cell type-specific enhancers. Nat Rev Mol Cell Biol. 2015;16:144-54.

12. Heyn H, Vidal E, Ferreira HJ, Vizoso M, Sayols S, Gomez A, et al. Epigenomic analysis detects aberrant super-enhancer DNA methylation in human cancer. Genome Biol. 2016;17:11.

13. Benetatos L, Vartholomatos G. Enhancer DNA methylation in acute myeloid leukemia and myelodysplastic syndromes. Cell Mol Life Sci. 2018;75:1999-2009.

14. Javierre BM, Burren OS, Wilder SP, Kreuzhuber R, Hill SM, Sewitz $\mathrm{S}$, et al. Lineage-specific genome architecture links enhancers and non-coding disease variants to target gene promoters. Cell. 2016;167:1369-84.

15. Schwartzman O, Mukamel Z, Oded-Elkayam N, Olivares-Chauvet $\mathrm{P}$, Lubling Y, Landan G, et al. UMI-4C for quantitative and targeted chromosomal contact profiling. Nat Methods. 2016;13: 685-91. 